\title{
A IMPORTÂNCIA DA EDUCAÇÃO CONTINUADA NA SOCIALIZAÇÃO DO NOVO PROFISSIONAL DE ENFERMAGEM
}

The Importance of Continuing Education in Socialization of Young Professional Nursing

Mariana Marques ${ }^{1}$, Diane Furtado dos Santos ${ }^{1}$, Maria Elvira de Oliveira Petersen², Márcia Regina Fidauza ${ }^{3}$

1 Enfermeira, Universidade do Extremo Sul Catarinense - UNESC.

${ }^{2}$ Enfermagem, Universidade de Cruz Alta - UNICRUZ, especialista em Auditoria de Sistemas de Saúde e Enfermagem do Trabalho.

${ }^{3}$ Enfermeira, Universidade do Oeste Paulista - UNOESTE, especialista em Gestão Hospitalar e em Administração Hospitalar.

\section{Endereço para Correspondência:}

Mariana Marques

Rua Nereu Ramos, n.ำ 26, Centro, Blumenau, Santa Catarina, CEP: 89010-400, Tel.: (048) 9617-0556

E-mail:marianaa_marques@hotmail.com 


\title{
Resumo
}

Trata-se de uma pesquisa de abordagem qualitativa, descritivo-exploratória e de campo. O estudo foi desenvolvido em um hospital de grande porte da região do Vale do Itajaí, tencionando descrever a importância do Serviço de Educação Continuada na Socialização do Novo Profissional que ingressa na Instituição como Enfermeiro Assistencial I nas Unidades Clínicas de Internação, realizando os cuidados integrais ao paciente no modelo de atenção à saúde, através da interdisciplinaridade no processo de trabalho. O estudo observa a importância da Educação Continuada em Saúde na socialização e acolhimento do novo profissional enfermeiro, menciona as dificuldades enfrentadas ao serem inseridos no mercado de trabalho, a transição existente entre a vida acadêmica e a vida profissional, e evidencia que muito rapidamente estes adquirem independência e segurança para executar as atividades de enfermagem.

Palavras-chave: Educação Continuada; Educação em Enfermagem; Socialização.

\begin{abstract}
This is a qualitative research, descriptive and exploratory field. The study was developed in a large hospital in the Itajai Valley region, intending to describe the importance of Continuing Education Service in the Socialization of New Professional who joins the institution as Nurse Care I in units Inpatient clinics, performing the comprehensive care to patient in the health care model through the interdisciplinary work process. The study notes the importance of Continuing Education in Health in socialization and acceptance of new professional nurse, mentions the difficulties faced when inserted in the labor market, the existing transition between academic life and professional life, and shows that very quickly they acquire independence and security to perform nursing activities.
\end{abstract}

Keywords: Continuing Education; Nursing Education; Socialization. 


\section{INTRODUÇÃO}

Os resultados obtidos através da socialização dos valores e normas profissionais e a experiência educacional em enfermagem envolve mais do que um corpo de conhecimentos científicos e a aquisição de habilidades para cuidar do paciente, indicando que os profissionais são influenciados pelo tempo de permanência na instituição, que os leva a diferenças de valores, normas e comportamentos profissionais ${ }^{1}$.

Os enfermeiros, durante todo o seu processo de formação, são introduzidos a um papel profissional idealizado, priorizando a interdisciplinaridade através do cuidado individualizado aos pacientes, com base em conhecimentos científicos, como a sua principal atividade profissional. No entanto, ao se inserirem no âmago de uma organização, deparam-se com a necessidade de assumir diversas tarefas e funções. Dessa forma, o reconhecimento das atividades desenvolvidas pelos enfermeiros no cotidiano das instituições é de fundamental importância, visto que possibilita visualizar mais claramente as lacunas existentes no seu trabalho e, consequentemente, auxiliar na delimitação das suas funções. Além disso, pode trazer elementos que possam contribuir para um desempenho mais criativo, inovador, participativo, mais próximo do paciente e do profissional e que favoreça o conhecimento das reais necessidades dos pacientes ${ }^{2}$.

Nas últimas décadas, houve um aumento da oferta de trabalho para os enfermeiros devido ao crescimento de cargos públicos, decorrentes da municipalização dos serviços de saúde após a Reforma Sanitária. Nesse contexto, houve uma necessidade da formação de mais profissionais da área, levando a um aumento da oferta de cursos de graduação em enfermagem, e consequentemente, aumento do número de profissionais formados. Atualmente, o mercado de trabalho para os enfermeiros dispõe de um elevado número de enfermeiros, o que proporciona aos empregadores o privilégio da escolha por profissionais melhor qualificados e com experiência na sua área de formação ${ }^{3}$.

Levando em consideração a complexidade das funções do profissional enfermeiro e as perspectivas a ele consignadas, a inserção no mercado de trabalho deste profissional sem experiência é vista com apreensão pelo mesmo, uma vez que 
se exige um conhecimento diversificado para superar a falta de experiência e enfrentar a competitividade 4 .

Muitos enfermeiros sentem-se inseguros ao ingressar no mercado de trabalho, visto que a graduação prepara o futuro profissional baseada no cuidado idealizado, o que pode divergir muito com a prática, considerando as dificuldades encontradas nos atuais ambientes profissionais de saúde. Além deste fator, enfatizase também que o ensino teórico de enfermagem deve ter uma estreita relação com a técnica em enfermagem, o que facilita que o futuro profissional possa ter maior conhecimento prático e menos receio em ingressar na profissão. Considera-se também o fato de as escolas de enfermagem atribuírem maior ênfase no ensino da área da saúde pública, influenciadas pelas políticas públicas de saúde vigentes, o que gera um sentimento de inaptidão destes profissionais para atuar ${ }^{4}$.

No processo de inserção de um enfermeiro no mercado de trabalho, a maneira como o mesmo é acolhido e socializado ao novo meio é imprescindível para sua adaptação e desenvolvimento. É natural que o novo profissional de enfermagem sinta-se inseguro no início da sua carreira perante a complexidade das suas atribuições, diante disso, é relevante que o mesmo passe por um processo de socialização com o novo ambiente profissional ${ }^{3}$.

O enfermeiro tem como uma de suas atribuições, liderar uma equipe de enfermagem, porém, sabe-se das dificuldades encontradas por estes profissionais ao lidarem com suas responsabilidades no início da carreira, sendo que coordenar uma equipe exige vasto conhecimento e estratégias de liderança que precisamos aprimorá-las ao longo do tempo. Levando em consideração este aspecto, a instituição abordada neste estudo buscou por estratégias para minimizar os efeitos negativos da falta de experiência na inserção do novo profissional de enfermagem no mercado de trabalho, oferecendo a oportunidade de trabalho para estes profissionais, com o intuito de contribuir para o seu desenvolvimento na enfermagem e aprimorar o cuidado aos pacientes, prezando por uma assistência baseada na interdisciplinaridade.

A nomenclatura de Enfermeiro Assistencial I foi atribuído ao profissional que está adentrando na instituição, e que muitas vezes é aquele profissional que não tem experiência, sendo esta sua primeira oportunidade de trabalho como 
enfermeiro. Suas atribuições serão os cuidados integrais ao paciente, e a execução de procedimentos privativos do enfermeiro, sendo este profissional acompanhado pelo serviço de Educação Continuada até que se perceba que o mesmo já pode exercer suas atividades sozinho. A instituição oferece a oportunidade de promoção para estes profissionais, abrindo processos seletivos internos para promoção de cargos, confiando aos mesmos a atribuição de Enfermeiro Assistencial II, que realiza as atividades de maior complexidade e auxilia o supervisor na coordenação de um determinado setor.

$O$ acolhimento aos profissionais de enfermagem que ingressam em uma instituição é de grande relevância para seu desenvolvimento, visto que o faz sentirse mais seguro para expor suas dúvidas e minimizar suas dificuldades. O profissional que é bem acolhido desempenha seu trabalho com maior autonomia, pois pode contar com 0 apoio da equipe e de outros profissionais que estão dispostos a contribuir para seu desenvolvimento ${ }^{5}$.

Muitas instituições têm buscado o Serviço de Educação Continuada como estratégia para qualificar seus profissionais, contudo, é necessário o entendimento de que ela pode ter uma amplitude muito maior do que apenas o aperfeiçoamento técnico, através de uma mudança de paradigmas e reafirmação de valores, sempre inserida em um contexto coletivo ${ }^{3}$.

O serviço de Educação Continuada da instituição abordada neste estudo conta atualmente com quatro enfermeiras que trabalham juntamente com a direção de enfermagem. Este serviço tem uma história recente, porém com um papel atual importante para os profissionais, que nos últimos anos vem recebendo treinamentos práticos, atualizações de conteúdos relacionados a enfermagem acerca da assistência, e o acompanhamento das atividades de enfermagem no âmbito hospitalar, assim como socializar, acolher e preparar o profissional Enfermeiro Assistencial I que está iniciando na instituição. O período de acompanhamento dos novos profissionais está relacionado com as necessidades e o desenvolvimento de cada um, respeitando o tempo de adaptação de cada profissional.

Os estudos realizados sobre esta temática ajudam a esclarecer as atividades desempenhadas pelos enfermeiros, contudo, essas atividades precisam ser constantemente investigadas, pois a realidade é dinâmica, as crenças e valores 
da profissão sofrem mudanças ao longo do tempo, exigindo o redimensionamento das competências e responsabilidades profissionais. Assim, este estudo busca identificar e analisar a socialização dos novos enfermeiros ao realizar atividades desempenhadas pelos enfermeiros assistenciais I nas unidades clínicas de internação, a fim de proporcionar informações sobre o papel desempenhado por esse profissional que possam contribuir para a melhoria da organização dos serviços de enfermagem.

Com o intuito de desvelar a socialização do profissional de enfermagem que ingressa em uma instituição hospitalar, tem-se como objetivo principal:

- Identificar qual a contribuição da Educação Continuada no acolhimento e socialização do novo profissional enfermeiro, que inicia sua atuação como Enfermeiro Assistencial I.

\section{MÉTODO}

O estudo busca conhecer o papel da Educação Continuada em Saúde na socialização e acolhimento do novo profissional enfermeiro que busca a oportunidade de ingressar no mercado de trabalho, expandindo o modelo dos enfermeiros assistenciais I, que desenvolvem atividades de enfermagem em unidades clínicas, realizando os cuidados integrais ao paciente, no modelo de atenção à saúde, através da interdisciplinaridade no processo de trabalho.

Trata-se de uma pesquisa de abordagem qualitativa, descritivoexploratória e de campo. O estudo foi desenvolvido em um hospital de grande porte da região do Vale do Itajaí. Fizeram parte da pesquisa trinta Enfermeiros Assistenciais I das unidades clínicas de internação que ingressaram na instituição com acompanhamento da Educação Continuada durante o período necessário para sua adaptação.

O procedimento de levantamento de dados iniciou com a aprovação do projeto de pesquisa pela gerência acadêmica da instituição. Posteriormente, realizou-se busca ativa pelos profissionais e aplicação de um questionário, que 
possibilitou a análise dos dados obtidos.Para a realização da pesquisa, os sujeitos assinaram um Termo de Consentimento Livre e Esclarecido, o qual assegura sigilo da identidade dos participantes.

A análise e interpretação dos dados qualitativos foi realizada baseando-se no método proposto por Minayo, através da categorização dos dados, através da ordenação, classificação e analise dos dados pesquisados. As categorias são empregadas para estabelecer classificações, significa agrupar elementos, ideias ou expressões em torno de um conceito capaz de abranger tudo que pode ser utilizado em qualquer tipo de análise em pesquisa qualitativa ${ }^{6}$.

\section{ANÁLISE DOS DADOS}

Os enfermeiros entrevistados foram nomeados com a letra $E$, seguido de um número para preservar a identidade dos sujeitos. Participaram do estudo trinta enfermeiros assistenciais I.

Dos entrevistados, vinte e sete são do sexo feminino e três do sexo masculino, a média de idade foi de vinte e oito anos, com uma média de dois anos e seis meses de formação na graduação em enfermagem, dos quais seis já tinham curso técnico em enfermagem, quinze afirmaram ser sua primeira oportunidade de trabalho na área da enfermagem. Através da análise dos dados obtidos com as entrevistas, foi possível estabelecer três categorias, sendo elas: Dificuldades enfrentadas para ingressar no mercado de trabalho; Acolhimento e socialização valorização do novo profissional; Educação Continuada e equipe de enfermagem como suporte para o novo profissional.

$\mathrm{Na}$ sequência, relatamos a categorização estabelecida com a descrição de falas de alguns sujeitos da pesquisa.

\section{Categoria 1: Dificuldades enfrentadas para ingressar no mercado de trabalho}


O processo de transição entre a vida acadêmica e a vida profissional é algo desafiador para os novos profissionais, algo que gera perspectivas e inseguranças em relação ao mercado de trabalho ${ }^{7}$.

Concomitantemente às perspectivas do novo profissional, lidamos com as exigências complexas do atual mercado de trabalho, que valoriza a competitividade e profissionais mais habilitados e capacitados. Portanto, a falta de experiência profissional é algo relevante e, em geral, torna-se uma dificuldade neste processo. $\mathrm{Na}$ sequência seguem algumas considerações dos sujeitos da pesquisa.

[...] procurei muito por uma oportunidade de emprego na minha cidade antes de ingressar aqui na instituição, porém, o mercado de trabalho ainda está muito fechado para os novos profissional (E4).

O mercado de trabalho moderno conta com um número considerável de profissionais de enfermagem, o que permite às empresas e empregadores 0 privilégio da escolha por profissionais mais capacitados, com melhor formação e maior experiência na área ${ }^{3}$.

De forma geral, percebe-se a queixa em relação à falta de oportunidades para o profissional inexperiente, que são considerados profissionais sem notáveis habilidades, o que gera desmotivação aos sujeitos.

[...] a maioria das empresas exige experiência, mas não dão oportunidade da gente se desenvolver como profissional (E10).

A qualidade profissional do enfermeiro frequentemente tem sido associada às habilidades técnicas, que consiste na destreza e agilidade para realização dos procedimentos de enfermagem, contudo, fica em segundo plano a capacidade do profissional de utilizar e integrar seus conhecimentos teóricos com as situações reais do cotidiano, com base em evidências científicas. Neste contexto, o novo profissional de enfermagem não tem oportunidades de demonstrar seus conhecimentos e habilidades devido à falta de experiências anteriores ${ }^{3}$.

É importante apontar que não só o mercado de trabalho pode contribuir para uma maior aceitação do novo profissional através de diferentes ideologias, mas que as escolas formadoras também têm uma importante atribuição neste contexto. Ainda que a academia tenha a responsabilidade do ensino do que é preconizado 
como ideal, é relevante também a associação deste aprendizado com as ações reais da prática, tornando o profissional recém-formado mais próximo do mercado de trabalho ${ }^{3}$.

É comum que no início da carreira os profissionais apresentem dificuldades, mas é notório que após um tempo de adaptação eles têm uma grande evolução em relação à realização das técnicas de enfermagem e à sua postura como enfermeiros. É relevante que eles se sintam uma peça importante no que diz respeito ao cuidado com o paciente e como parte da equipe de enfermagem, para que possam desenvolver sua autonomia e aprimorar habilidades.

\section{Categoria 2: Acolhimento e socialização - valorização do novo profissional}

A dificuldade de ingressar no mercado de trabalho, o processo de transição entre o mundo acadêmico e o profissional, as expectativas sobre suas próprias capacidades profissionais, a ansiedade motivada pelo desconhecimento são algumas situações que podem gerar insegurança e incertezas no processo de inserção do novo profissional em uma organização/instituição. A integração ou socialização de enfermagem pode amenizar esses efeitos negativos da chegada do profissional ao novo ambiente de trabalho, proporcionando um bom relacionamento entre os sujeitos, através do acolhimento e acompanhamento ${ }^{8}$.

O acolhimento no novo ambiente de trabalho é um fator imprescindível para uma boa adaptação e desenvolvimento profissional, bem como para um bom funcionamento dos serviços. Os novos profissionais que ingressam no mercado de trabalho têm a necessidade de sentir-se familiarizados com o ambiente e a equipe, para que possam ter mais autoconfiança nas suas ações ${ }^{5}$.

Através das falas, foi possível denotar ainda a relevância do acolhimento como estratégia inicial para o desenvolvimento do profissional:

[...] o acolhimento me deu a sensação de que realmente sou importante para a instituição (E1).

"O profissional que é bem acolhido sente-se seguro para realizar seu trabalho e, também, buscar autonomia dentro do cenário prático em que está inserido" ${ }^{\prime 3}$. 
Alguns entrevistados enfatizaram ainda mais resultados positivos da socialização neste processo:

[...] essa socialização faz todo o diferencial na admissão do novo profissional, porque com essa ação nós nos sentimos melhor recepcionados e, nos incentiva a nos esforçarmos para corresponder às expectativas (E3).

[...] achei a socialização da instituição bem acolhedora, é um incentivo a mais para nós que estamos chegando agora (E1).

Percebe-se que o acolhimento e acompanhamento do novo profissional pode impulsioná-lo a buscar por aperfeiçoamento, visto que o mesmo sente necessidade de apresentar resultados satisfatórios aos que estão the depositando confiança.

\section{Categoria 3: Educação Continuada e equipe de enfermagem como suporte para o novo profissional}

O conceito de Educação Continuada é antigo, porém recentemente é que tem sofrido reformulações em seu conceito que realmente condizem com seu valor perante a sociedade. Atualmente, a Educação Continuada é considerada um "processo educativo formal ou informal, dinâmico, dialógico e contínuo, de revitalização pessoal e profissional, de modo individual e coletivo, buscando qualificação, postura ética e reformulação de valores", o qual pode contribuir para uma relação integradora entre os sujeitos envolvidos e a instituição ${ }^{9}$.

Tratando-se de saúde, e mais especificamente da enfermagem no âmbito hospitalar, a Educação Continuada pode ser um modo de alcançar uma assistência de qualidade, pois engloba programas de ensino-aprendizagem que proporcionam aos profissionais o desenvolvimento de suas habilidades e pensamento holístico e crítico, proporcionando um cuidado voltado para a interdisciplinaridade. Desta forma, o objetivo da Educação Continuada é promover o crescimento profissional e pessoal, analisar e desenvolver competências individuais e coletivas, priorizando a qualidade da assistência de enfermagem ${ }^{1}$.

$\mathrm{Na}$ referida instituição em que foi realizado o estudo, a Educação Continuada tem como uma das atribuições o acompanhamento dos novos 
profissionais, além do aperfeiçoamento da equipe de enfermagem. Atualmente, ao ingressar na instituição, os novos profissionais participam de uma socialização no primeiro dia em que são apresentadas normas gerais sobre o funcionamento do hospital, no segundo dia participam da socialização de enfermagem, em que são apresentadas normas e rotinas do departamento. A partir do terceiro dia, os novos profissionais são acompanhados nas unidades clínicas de internação, onde são apresentadas as rotinas e os mesmos conseguem ter maior proximidade com a prática por meio da assistência de enfermagem ao paciente, sempre supervisionados pelas enfermeiras da Educação Continuada.

[...] recebi acompanhamento das enfermeiras da Educação Continuada. Tive duas semanas de socialização, que me ajudaram a conhecer melhor o hospital e também a realizar procedimentos em que tinha dúvidas (E7).

[...] o treinamento me direcionou para atuar no setor, a prática vem com a rotina diária, mas o treinamento direciona sobre as rotinas e política hospitalar (E13).

O tempo mínimo de acompanhamento da Educação Continuada a estes novos profissionais nas unidades de internação é de uma semana, podendo se estender por quanto tempo for necessário, dependendo do desenvolvimento de cada profissional, respeitando o tempo de adaptação de cada um e suas individualidades.

[...] o tempo depende de cada profissional, pois cada um tem seu tempo de aprendizado, para mim foi o suficiente (E1).

[...] quando eu fiz achei que foi o tempo necessário para conhecer as rotinas e normas da instituição (E9).

[...] acredito que seja tempo suficiente, porém, cada indivíduo tem o seu tempo para aprender, alguns mais rápido ou mais demorado (E20).

É possível neste período a observação deste profissional nos seus diversos aspectos, portanto, avalia-se principalmente suas maiores dificuldades e busca-se por estratégias para a otimização do seu desenvolvimento profissional. Durante esta etapa do acompanhamento consegue-se ter maior proximidade com os 
profissionais, criando um vínculo entre os sujeitos e proporcionando segurança aos novos profissionais.

Além do acompanhamento pela equipe da Educação Continuada no momento em que o profissional ingressa na instituição, é necessário também que haja uma mudança nos padrões de toda a equipe de enfermagem, pois além do aperfeiçoamento técnico do novo profissional, é importante que ele se sinta inserido no contexto de equipe. Ao ser alocado no seu setor destino, o profissional passa um período de adaptação, e a equipe pode ser uma facilitadora de todo este processo ${ }^{3}$.

Através das falas, foi observada a importância da equipe de enfermagem na inserção, adaptação e desenvolvimento destes profissionais:

[...] o tempo de acompanhamento acredito que seja o suficiente, após este tempo o profissional pode se reportar ao seu coordenador e sua equipe (E5).

[...] Não tive muita dificuldade de adaptação, pois quando entrei na instituição, a supervisora do setor e os meus colegas sempre me acompanharam e me ajudaram no que precisava isso foi muito importante (E16).

Embora se saiba sobre a relevância da presença e apoio da Educação Continuada e da equipe de enfermagem na admissão do profissional na instituição, é fundamental o entendimento de que a enfermagem é uma profissão que necessita de aprimoramento contínuo, de evolução constante, levando em consideração o real conceito de Educação Continuada, como citaram alguns entrevistados:

[...] agora me sinto segura nas atividades, pois já tenho um tempo de trabalho, e os treinamentos ajudam a melhorar mais e mais, porém, sinto que ainda há muito que aprender (E22).

[...] Todos os dias aprendo algo novo, e acredito que enfermagem é isso, um aprendizado a cada dia (E23).

O estudo demonstrou ainda a consciência dos profissionais em relação a importância de seu próprio empenho para um bom desenvolvimento na instituição, 
trazendo para si mesmo parte da responsabilidade de uma boa adaptação e inserção na equipe de enfermagem:

[...] primeiramente o interesse deve partir do profissional em aprender e a Educação Continuada dá uma estrutura de reforço para complementar o meu aprendizado (E19).

[...] a intenção do acompanhamento é excelente, mas tudo depende do profissional, do interesse que ele tem de agregar conhecimento (E21).

Sabe-se das dificuldades encontradas pelos profissionais de adaptar-se a uma nova rotina e evoluir profissionalmente e constantemente, sendo assim, o trabalho da Educação Continuada, pode contribuir de forma positiva neste processo. Através da interdisciplinaridade, é possível aprimorar os conceitos técnicoscientíficos dos profissionais, de forma que se viabilize uma avaliação e cuidado amplo do paciente, levando em consideração suas individualidades em diversos aspectos.

\section{CONCLUSÃO}

O presente estudo demonstra que são muitas as dificuldades enfrentadas pelos novos profissionais ao serem inseridos no mercado de trabalho. Portanto, vêse a necessidade de proporcionar ao profissional um acolhimento e acompanhamento a fim de mitigar os efeitos negativos de ingressar em um novo ambiente de trabalho. Percebe-se que nem sempre o profissional entra no mercado de trabalho com grandes habilidades técnicas, pois existe uma transição entre a vida acadêmica e a vida profissional que gera expectativas e inseguranças, e que deve ser respeitado o tempo que cada um necessita para sua adaptação.

Ao trazer as experiências do novo profissional que está ingressando no ambiente de trabalho, percebe-se que é imprescindível para adaptação e desenvolvimento profissional, que ele tenha um bom acolhimento por parte da educação continuada e toda equipe de enfermagem, tornando possível uma socialização coletiva eficaz, que diminui efeitos negativos e proporciona bons 
relacionamentos entre os sujeitos, pois a confiança depositada sobre os mesmos os impulsiona a ficarem mais autoconfiantes a sentir-se parte da equipe.

Acredita-se o objetivo principal deste estudo tenha sido alcançado, pois evidenciou-se através das falas dos participantes que o serviço da Educação Continuada tem contribuído positivamente para este processo de acolhimento e socialização na inserção dos novos profissionais designados Enfermeiros Assistenciais I na instituição. Evidencia-se que estes muito rapidamente adquirem independência e segurança para executar as atividades de enfermagem. Cabe aqui ressaltar como sugestão o aprimoramento do Serviço de Educação Continuada e da equipe de enfermagem, buscando sempre a interdisciplinaridade da assistência de enfermagem.

\section{REFERÊNCIAS}

1. Pedott K. A importância da educação continuada na instituição hospitalar. Rev. Contestado. 2012;1(1):22-34.

2. Costa RA, Shimizu HE. Atividades desenvolvidas pelos enfermeiros nas unidades de internação de um hospital-escola. Rev. Latino-Am. Enfermagem. 2005;13(5):654-62.

3. Jesus BH, Gomes DC, Spillere LBB, Prado ML, Canever BP. Inserção no mercado de trabalho: trajetória de egressos de um curso de graduação em enfermagem. Esc. Anna Nery. 2013;17(2):336-45.

4. Carrijo CIS, Bezerra ALQ, Munari DB, Medeiros M. A empregabilidade de egressos de um curso de graduação em enfermagem. Rev. Enferm. UERJ. 2007;15(3):356-63. 
5. Silva DGV, Souza SS, Trentini M, Bonetti A, Mattosinho MMS. Os desafios enfrentados pelos iniciantes na prática de enfermagem. Rev. EscEnferm. USP. 2010;44(2):511-6.

6. Minayo MCS. Pesquisa Social: teoria, método e criatividade. $21^{1 \mathfrak{a}}$ edição. Petrópolis: Vozes, 2009.

7. Colenci R, Berti HW. Formação profissional e inserção no mercado de trabalho: percepções de egressos de graduação em enfermagem. Rev. Esc. Enferm. USP. 2012;48(1):158-66.

8. Machado G, Moretto Neto L, Bernardini IS. Proposta de um Programa de Acolhimento dos Novos Trabalhadores da Secretaria Municipal de Saúde de Florianópolis, em Santa Catarina. Coleção Gestão da Saúde Pública. 2012;10(1):50-78.

9. Nietsche EA, Backes VMS, Ferraz F, Loureiro L, Schmidt SMS, Noal HC. Política de educação continuada institucional: um desafio em construção. Rev. Eletr. Enf. 2009;11(2):341-8. 